\title{
Vector Network Analysis Using a One-Path, Frequency-Multiplied Photonic Link
}

\author{
Dong-Joon Lee ${ }^{1} \cdot$ Jae-Yong Kwon $^{1} \cdot$ Jin-Seob Kang $^{1} \cdot$ John F. Whitaker $^{2}$
}

\begin{abstract}
A simplified, practical vector network analyzer (VNA) that uses mature radio-over-fiber technology has been designed and demonstrated. The measurement concept allows the full $S$-parameters of a microwave device (or antenna) to be obtained while minimizing the detrimental effects of electrical cables, which are replaced with a photonic link. A variety of high-frequency light modulation schemes with frequency sweeping capabilities are presented to realize a one-path (single, forward), frequency-multiplied optical link for VNA applications. Using the photonic one-path link, full two-port $S$-parameters have been extracted based on five-term error modeling, which has half the error terms compared with the standard duplex configuration. The $S$-parameters of a microwave filter and antenna measured using frequency-multiplied optical links are found to be in good agreement with those obtained using a conventional VNA.

Key words : S-Parameter Measurement, One-Path Two-Port Calibration, Radio-Over-Fiber (RoF).
\end{abstract}

\section{Introduction}

The impedance or transfer function performance of microwave devices has generally been evaluated by measuring scattering $(S)$-parameters over their operating frequency range. To obtain the $S$-parameters as arrays of complex numbers, commercial vector network analyzers (VNAs) have been widely employed. Although the use of an electrical network analyzer has become a standard method for impedance measurement over the past four decades, there are some remaining challenges due to the lossy and noise-susceptible electrical embodiment, especially for high frequency applications. For instance, in measuring an insertion/return loss of an antenna in a huge measurement chamber or across potentially long distances in open-area test sites, the cost of lengthy cables and extra components, such as amplifiers, used to compensate the cable losses become a concern. Moreover, the intrinsic characteristics and electromagnetic compatibility performance of a device under test (DUT) - and in particular antennas - are often affected by the presence and even arrangement of the cascaded metallic cables [1 3].

To counteract these detrimental influences, mature optical telecommunications techniques have been adopted over the last decade [4]. For instance, laser diode light, which serves as an optical carrier, is electro-optically modulated with microwave sidebands. The sidebands can be optically delivered over fairly long distances through optical media and finally demodulated to obtain the electrical version of signals for microwave applications. Concerns with the use of metallic cables are significantly mitigated by replacing the majority of electrical interconnections with optical links. Besides optical links having immunity to electromagnetic interference, the bandwidth (BW) of microwave networks can be significantly enhanced using nonlinear optical light modulation techniques [5 14]. This is due to the rapid development of high-frequency light modulation schemes that can accommodate an expanded number of channels with higher data transmission rates.

In this paper, we have achieved what we believe to be the first implementation of a VNA using a frequencymultiplied optical link system. The performances of our system using both fundamental and frequency- doubled optical links are cross-checked, with one-port and twoport calibrations, versus a conventional VNA. A frequencyquadrupling technique, its challenges, and realization are also discussed.

The photonic single-forward one-port VNA system with three-term error modeling is introduced in Section $\Pi$ and then the system is extended for the full two port $S$-parameters analysis adding two more error terms in Section III. Finally, the modified system using a downmixing technique for practical high-frequency antenna measurement is presented in Section IV.

\footnotetext{
Manuscript received September 2, 2010 ; revised October 29, 2010. (ID No. 20100902-029J)

${ }^{1}$ Center for Electromagnetics, Korea Research Institute of Standards and Science, Daejeon, Korea.

${ }^{2}$ Center for Ultrafast Optical Science and Department of Electrical Engineering and Computer Science University of Michigan, Michigan, USA.

Corresponding Author : Dong-Joon Lee (e-mail : dongjoonlee@kriss.re.kr)
} 


\section{II . One-Port Calibration with a Photonic Link}

In most conventional electronic VNAs, the two ports of the instrument are bidirectional. Thus, each port can transmit and receive signals through common paths. Even for one-port measurements, the port is generally intended to transmit and receive power from an antenna (i.e., a reflection) or DUT. It is a challenge to share the common path in order to replace an electrical path with an optical link because the optical link going from the laser to the detector is inherently unidirectional. Therefore, another port is needed as a receiver port. Here, rather than using an expensive VNA that has the option to assign each port as a respective transmitter- or receiver-only port, we employed a discrete and cost-effective optical link configuration for one-port calibration.

Fig. 1 is the one-port calibration system with an optical link, consisting of $1,550 \mathrm{~nm}$ standard optical telecommunication parts. For conventional fundamental order amplitude modulation, the DC bias is set to the center of the cosine-squared modulation slope, and thus the light is linearly modulated in response to the electrically driven signals. The power level of the radio frequency (RF)-modulated light is adjusted to be approximately 1 $\mathrm{mW}$ to avoid photodiode saturation. The light is then demodulated through a photodiode and the demodulated RF components are transmitted from the 50- $\Omega$ output port of the diode to the electrical receiver system. This serves as a closely located virtual RF source for the electrical calibration system.

The RF input power is divided into two, with half being delivered to port 1 and half being used as a reference to resolve the relative amplitude and phase information at coupling ports $A$ and $R$. To calibrate port 1 as a virtually perfect reflectometer, we employed the

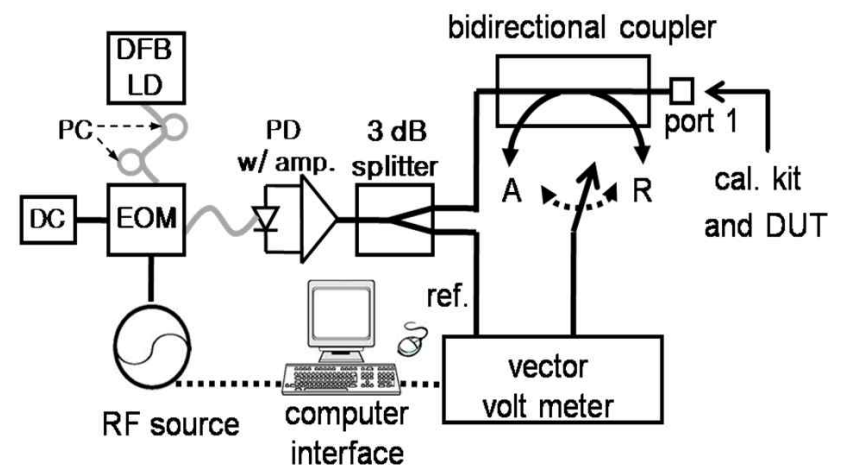

Fig. 1. One-port calibration system with optical link (DFB LD: distributed feedback laser diode; EOM: electro-optic modulator; PC: polarization controller; PD: photodiode). The gray and black lines are optical and electrical connections, respectively.

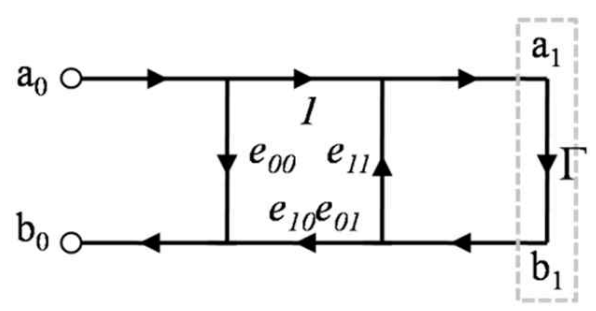

Fig. 2. Three-term error model for one-port calibration.

conventional three-term error model $[15,16]$. This model deals with three dominant and systematic error terms in an actual reflectometer, known as the directivity $\left(e_{00}\right)$, port match $\left(e_{11}\right)$, and reflection tracking $\left(e_{10} e_{01}\right)$ errors at the port; these terms are readily extracted using Eq. (1).

$$
\left[\begin{array}{c}
e_{00} \\
e_{11} \\
\Delta_{e}
\end{array}\right]=\left[\begin{array}{ll}
1 & \Gamma_{1} \Gamma_{m 1}-\Gamma_{1} \\
1 & \Gamma_{2} \Gamma_{m 2}-\Gamma_{2} \\
1 & \Gamma_{3} \Gamma_{m 3}-\Gamma_{3}
\end{array}\right]\left[\begin{array}{c}
\Gamma_{m 1} \\
\Gamma_{m 2} \\
\Gamma_{m 3}
\end{array}\right]
$$

where $\Delta_{e} \equiv e_{00} e_{11}-e_{10} e_{01}, \quad \Gamma_{i}$, and $\Gamma_{m i}(i=1,2,3)$ are known DUTs (such as calibration kits) and their measured reflection values, including errors, respectively.

The reflection values are expressed as $A_{\text {ref }} / R_{\text {ref }}(=\Gamma)$, where, in order to resolve the phase information, the subscript indicates that each $A$ and $R$ is the value with respect to the reference signal. We extracted the three error terms with an $\mathrm{N}$-type short-open-load (SOL) calibration kit (Agilent Technologies: 85054B) and measured three standard mismatch loads. The voltage standing wave ratios (VSWRs) for the standard loads we employed were 1.2, 1.5 and 1.75; such loads are commonly used to verify the performance of a VNA. Here, we used these reference loads to evaluate our one-port calibration system. Each error-compensated value (black lines: $1 \sim 1,000 \mathrm{MHz}$ ) in Fig. 3 agrees well with that measured with a commercial VNA (gray lines: $50 \sim 1,000$ $\mathrm{MHz}$ ). The bandwidth of our current system is $1 \mathrm{GHz}$, limited by our photodetector (New Focus: 1611-FC-AC) and read-out instrument (Agilent Technologies: 8508A). However, unlike most commercial VNAs, this type of homemade system readily covers lower bands down to $1 \mathrm{MHz}$ or even less by supplying an appropriate electrical source.

\section{Two-Port Calibration with Frequency-Folded Optical Link}

\section{3-1 Source Generation for Two-Port Calibration}

One of the modern trends of radio-over-fiber (RoF) technology is very high-speed light modulation, used in order to accommodate the exploding demand for more channels with faster rates. Multiplication of modulating 


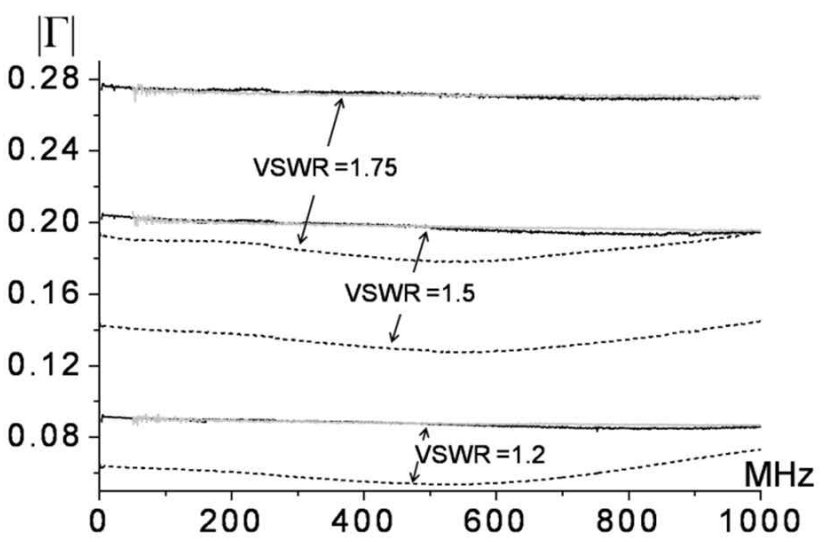

Fig. 3. Reflection coefficients of three standard mismatch loads measured using the photonic link network analysis system (black solid lines: calibrated; black dashed lines: uncalibrated) and a commercial VNA (gray lines: calibrated). All the data are measured with 1 $\mathrm{MHz}$ step resolution.

frequencies by enhancing the nonlinearity of electrooptic modulators (EOMs) has garnered great attention for several years $[5 \sim 14]$. The most common and straightforward type of frequency multiplication is doubling, which can be readily achieved by simply shifting the DC bias of an electro-optic modulator to one of its minimum (null) intensity transmission points [9, 10]. This scheme does not require modifying the optical link configuration in Fig. 1.

For higher even-order harmonic frequency generation, a second EOM is employed to boost the nonlinear modulation slope of the set of modulators. Numerous efficient methods have been reported for this process $[5 \sim 8$, $11 \sim 14]$. One simple and widely used frequency-quadrupling configuration is a cascaded arrangement, as shown in Fig. 4 [6, 13, 14]. The DC bias values for each EOM are generally set at null transmission points, as in the second-harmonic generation case, to suppress odd-order harmonics. To suppress undesired even-order harmonics (especially the second harmonic for quadrupling), the relative phase offsets to each EOM must be $\pm 90^{\circ}$. This quadrature phase condition can be realized by adjusting the RF phase for one EOM or the optical phase delay between the two EOMs.

Efficient frequency quadrupling can be successfully achieved by effective quadrature phase cancellation. However, since the phase relations are strongly related to the operating frequencies, such 'in-quadrature' conditions are usually met only at particular frequencies. For instance, Fig. 5 shows the evolution of the even-order harmonics with different phase conditions between the two modulators. In our setup, we used two identical RF cables and thus there is no electrical phase difference for the

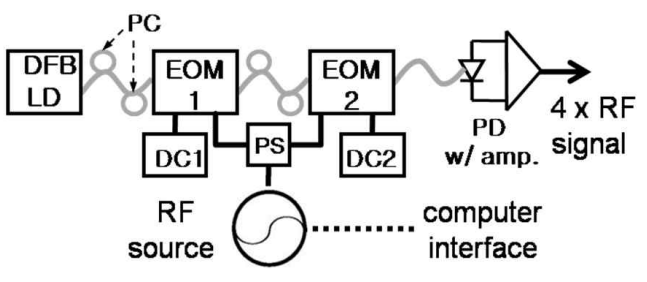

Fig. 4. Frequency-quadrupling configuration with cascaded electro-optic modulators (EOMs) (black lines: electrical connections; gray lines: fiber optic connections). (DFB LD: distributed feedback laser diode; PS: power splitter; PC: polarization controller; PD: photodiode).

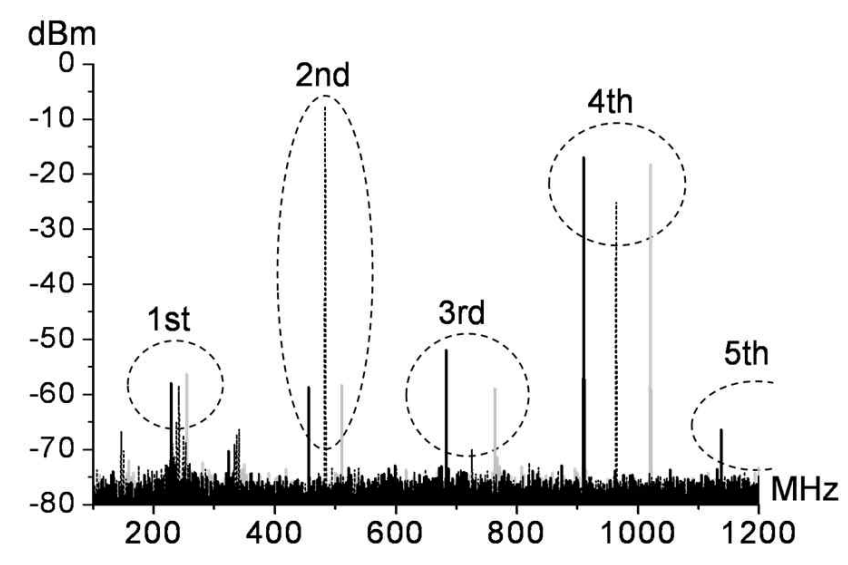

Fig. 5. Frequency-folded spectra by cascaded electro-optic modulators. The black and gray solid (and dashed) lines are for the in-(and out-of) quadrature condition for the fourth (and second) harmonic generations.

inputs to the EOMs. The only delay is the optical fiber delay between the EOMs. For example, the second-order components are best suppressed at $227.314 \mathrm{MHz}$ of $\mathrm{RF}$ feeding with $+13 \mathrm{dBm}$ (solid black spectra). The next second-order suppression occurs at $254.683 \mathrm{MHz}$, which corresponds to the next-order in-quadrature condition. On the other hand, the second-order components are maximized at the middle of the in-quadrature conditions, where the components constructively combine (i.e., outof-quadrature conditions).

Although this frequency-multiplying scheme can suppress undesired harmonics $30 \mathrm{~dB}$ below the multiplied signal (thus making it suitable for single-tone source generation), there is a distinct challenge to apply it as a tunable source having a fixed phase delay. It is possible to adjust a phase delay to maximize the quadrupling efficiency at certain frequencies by shifting an electrical or optical delay. Thus, we should technically be able to control the in-quadrature condition as the driving frequency changes. In practical situations, the $27.37 \mathrm{MHz}$ mode spacing between two in-quadrature conditions (= 


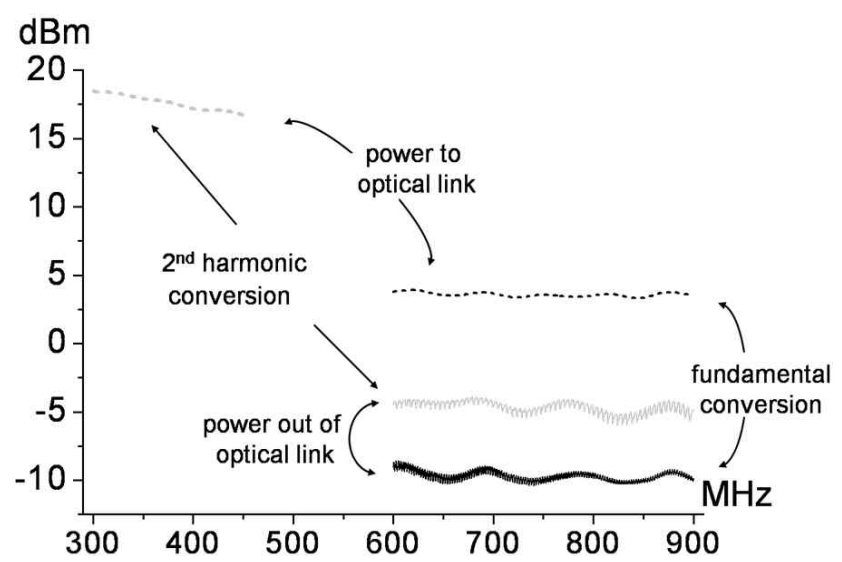

Fig. 6. Conversion efficiency of the RoF optical link. The black (or gray) dashed line is the driving power to an EOM and the black (or gray) solid line is the converted power (all data are measured with 0.2 $\mathrm{MHz}$ step resolution).

|254.683 227.314| MHz) corresponds to $36.536 \mathrm{~ns}$ (= $180^{\circ}$ phase shift). To compensate for such a mode phase drift, 5.48 meters of a controllable round-trip free-space delay path between EOMs is required. Even such a long optical path control would yield only $109.48 \mathrm{MHz}$ of fourth harmonic bands, which is inadequate for typical network analyzer applications. However, since this frequency-folding technique is advantageous for millimeter wave applications rather than $\sim \mathrm{GHz}$ ones, the optical delay scale will be reduced accordingly.

Here, we used fundamental and second-order harmonics of the EO-OE conversion scheme to create reliable frequency sweeping solutions. Using a single EOM, as in Fig. 1, the phase drifting problem associated with multiple EOMs is removed. The spectral power of electrical driving and the resulting power to the DUT are shown in Fig. 6 for the respective fundamental and second-order harmonic methods. The black (gray) dashed line is the driving power to an EOM and the black (gray) solid line is the EO-OE converted power that is to feed the DUT. The converted power is affected by the response of the EOM and photodiode, and the small ripples in the converted power can be reduced using an optical isolator. Although the EO-OE converted responses are less uniform than those of the system fed by a conventional RF source, it is not detrimental for $S$-parameter measurement because of the calibration procedure in the next section.

\section{3-2 Two-Port Calibration with Photonic Link}

In a calibration and measurement system, the RF calibration operation does not distinguish whether the incoming microwave signals are generated by a nonlinear

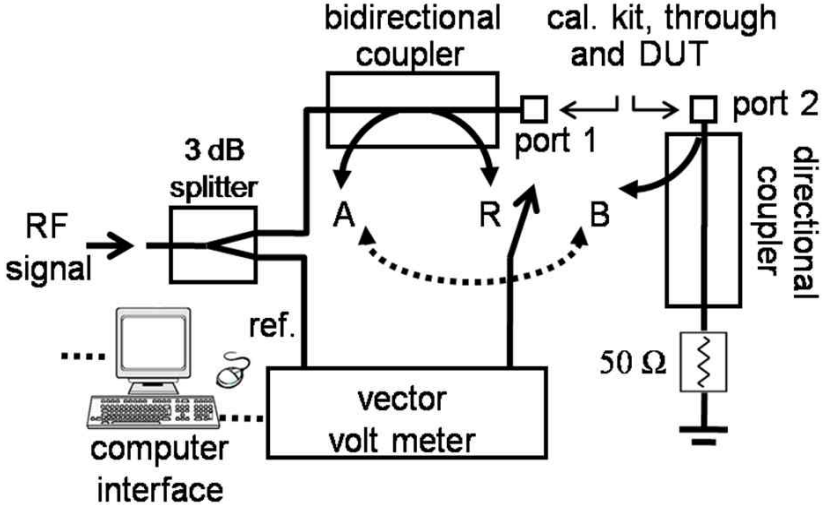

Fig. 7. Two-port VNA calibration system (unused ports need to be terminated with a $50 \Omega$ load).

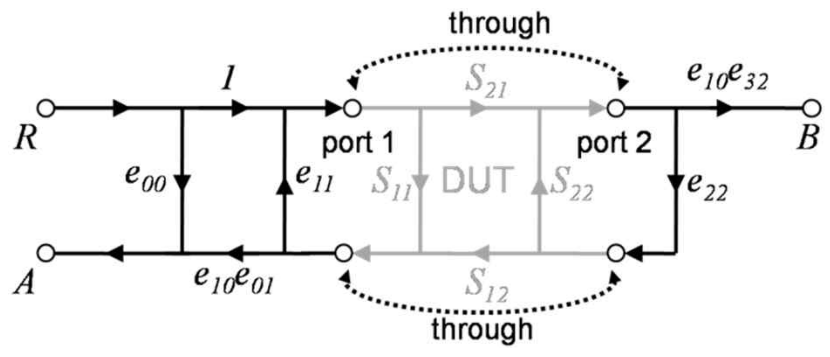

Fig. 8. Five-term error model for two-port calibration (a through is for $e_{10} e_{32}$ and $e_{22}$ ).

process or are delivered optically/electrically, as long as they maintain good spectral quality. Thus, we can simplify an optical link system as an RF source module and move on to the two-port calibration. The extended system based on Fig. 1 is presented in Fig. 7.

The conventional error model for calibrating the forward one-path, two-port system is a five-term error model $[15,16]$. With a second port (port 2), transmission tracking $\left(e_{10} e_{32}\right)$ and port 2 mismatch $\left(e_{22}\right)$ terms are added, as shown in Fig. 8.

The error terms associated with port $2\left(e_{10} e_{32}\right.$ and $\left.e_{22}\right)$ are extracted by a Thru (=through) calibration procedure. Measuring the referenced values at each coupling port, $R_{r e f}, A_{r e f}$, and $B_{r e f}$, then $e_{22}$ and $e_{10} e_{32}$, in terms of the raw transmission and reflection values and other errors, are expressed as Eq. (2).

$$
\begin{gathered}
e_{22}=\frac{\left(\frac{A_{\text {ref }}}{R_{\text {ref }}}\right)-e_{00}}{\left(\frac{A_{\text {ref }}}{R_{\text {ref }}}\right) e_{11}-\Delta_{e}}, \\
e_{10} e_{32}=\left(\frac{B_{\text {ref }}}{R_{\text {ref }}}\right)\left(1-e_{11} e_{22}\right)
\end{gathered}
$$

The five error terms enable the measured $S$-parameters $\left(S_{m}\right)$ to be transformed into those of the actual DUTs. 
In conventional duplex error-term modeling, the reverse error terms are also considered because most commercial networks switch the signal flow path during measurement for convenience. However, it is a challenge to replace partial electrical connections with an optical link and it becomes more costly to implement the bidirectional configuration. Thus, modifying the bidirectional ten-term error model for one-path forward modeling, the fully calibrated $S$-parameters with five error terms can be expressed as Eq. (3).

$$
\begin{aligned}
& S_{11}=D^{-1}\left[\left(\frac{S_{11 m}-e_{00}}{e_{10} e_{01}}\right)\left(1+\left(\frac{S_{22 m}-e_{00}}{e_{10} e_{01}}\right) e_{11}\right)-\left(\frac{S_{21 m} S_{12 m} e_{22}}{\left.e_{10} e_{32}\right)^{2}}\right)\right] \\
& S_{21}=D^{-1}\left[\frac{S_{21 m}}{e_{10} e_{32}}\left(1+\left(\frac{S_{22 m}-e_{00}}{e_{10} e_{01}}\right)\left(e_{11}-e_{22}\right)\right)\right] \\
& S_{12}=D^{-1}\left[\frac{S_{12 m}}{e_{10} e_{32}}\left(1+\left(\frac{S_{11 m}-e_{00}}{e_{10} e_{01}}\right)\left(e_{11}-e_{22}\right)\right)\right] \\
& S_{22}=D^{-1}\left[\left(\frac{S_{22 m}-e_{00}}{e_{10} e_{01}}\right)\left(1+\left(\frac{S_{11 m}-e_{00}}{e_{10} e_{01}}\right) e_{11}\right)-\left(\frac{S_{21 m} S_{12 m} e_{22}}{\left(e_{10} e_{32}\right)^{2}}\right)\right]
\end{aligned}
$$

where

$$
D=\left[1+\left(\frac{S_{11 m}-e_{00}}{e_{10} e_{01}}\right) e_{11}\right]\left[1+\left(\frac{S_{22 m}-e_{00}}{e_{10} e_{01}}\right) e_{11}\right]-\left[\frac{S_{21 m} S_{12 m} e_{22}^{2}}{\left(e_{10} e_{32}\right)^{2}}\right]
$$

Note that the calibration of each $S$-parameter requires all four measured $S$-parameters with five forward error terms. Measuring $A_{\text {ref }} / R_{\text {ref }}$ and $B_{r e f} / R_{\text {ref }}$ with a DUT yields $S_{11 m}$ and $S_{21 m}$ and reversing the DUT's ports gives $S_{22 m}$ and $S_{12 m}$, respectively.

To try out the calibration process initially, the full $S$-parameters of a flat-top band-pass filter that had a 3 $\mathrm{dB}$ bandwidth of $742.6 \sim 777.6 \mathrm{MHz}$ were measured (Fig. 9). The filter was characterized using the fundamental and frequency-doubling optical links, and each result was compared with those of a VNA.

The two EO-OE converted swept band feeds were respectively used to analyze the DUT and their transmission and reflection values were compared using a commercial VNA (Agilent Technologies, E8361C). The reflection curves agree well, except at reasonably low levels around approximately $-30 \mathrm{~dB}$. Also, the transmission plots are indistinguishably matched, except at their noise floors, which are fundamentally determined by the capability of the vector read-out instrument. The noise level could be lowered by employing a lock-in detection scheme, which has ultra-narrow band-pass capability around the signal of interest.

\section{Application of Optical Link to High-Frequency Antennas}

Although the results of Section III confirm that an op-

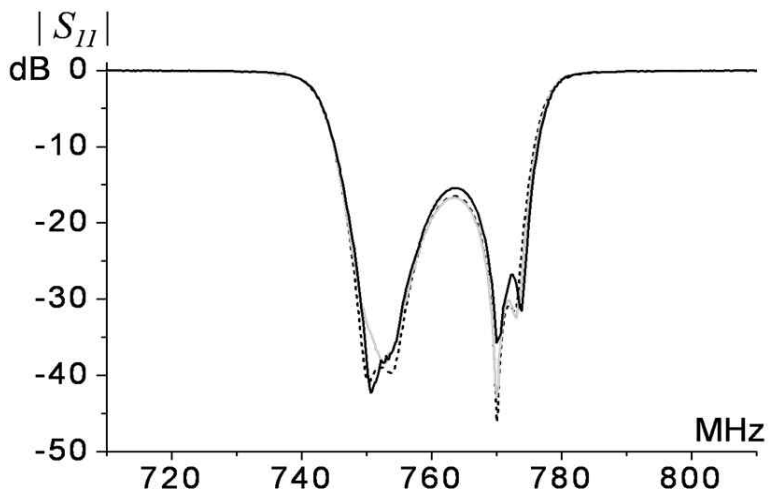

(a)

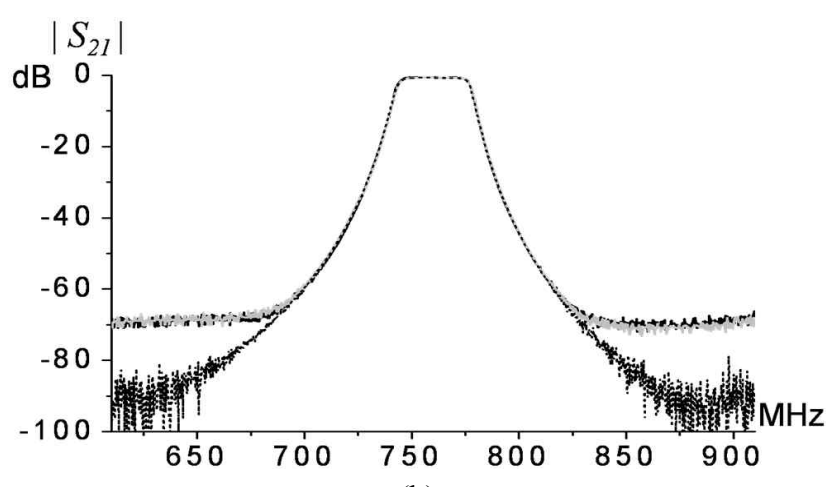

(b)

Fig. 9. $S$-parameters of a band-pass filter (a) Reflection (b) Transmission (black solid lines: fundamental optical link; gray solid lines: frequency doubling optical link; black dashed lines: conventional VNA). The $\left|S_{21}\right|$ plots within the pass band are indistinguishably overlapped.

tical link can provide the same performance as conventional methods in sub-GHz bands, an optical link not only would be more advantageous for use at higher frequencies, but it could also overcome the electronic limits of conventional methods. The relatively low $1 \mathrm{GHz}$ bandwidth can be extended considerably to the higher bands using a frequency-multiplied photonic local oscillator (LO) sideband on the optical carrier beam and the electrical heterodyne down-conversion scheme [10].

Fig. 10 shows the one-port calibration system using the down-converting optical link. This is basically an extension of the configuration in Fig. 1, with the addition of a frequency-multiplied LO light-modulation link to down-mix the signal frequency to an intermediate frequency (IF) within the bandwidth of an RF lock-in amplifier. Each link consists of a $15 \mathrm{GHz} \mathrm{BW}$ amplitude modulator (JDSU: OC-192) and a $20 \mathrm{GHz}$ BW photodetector (EM4 inc: EM169) to be used for the EO-OE conversion. The RF signal link also uses the frequencydoubling scheme of Ref 10 to expand the system fre- 


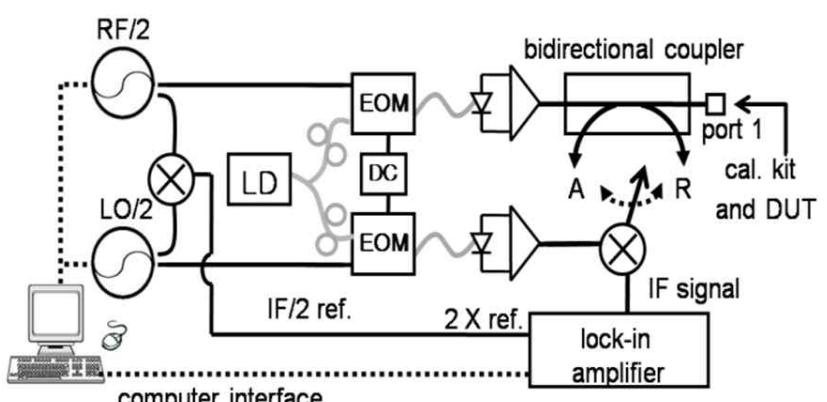

Fig. 10. One-port calibration system with heterodyning optical link (the gray and black lines are optical and electrical connections, respectively); EOM is a standard telecommunications electro-optic modulator, DC is the dc bias for the modulators, and LD is a laser diode.

quency response above $15 \mathrm{GHz}$, for upper- $\mathrm{Ku}$ and $\mathrm{K}$ band applications, so that nearly no components or instruments having capabilities past $15 \mathrm{GHz}$ are needed.

To demonstrate the calibration procedure with the photonic link VNA at these higher frequencies, the return loss was measured on a $4 \times 4$ antenna array that had previously been observed to have multiple resonance frequencies in the $\mathrm{Ku} / \mathrm{K}$ band region [18]. The signal source, at a frequency of RF/2, was swept between 8 $10 \mathrm{GHz}$ with $3 \mathrm{MHz}$ steps so as to yield a $16 \sim 20 \mathrm{GHz}$ DUT input range, and the $\mathrm{LO} / 2$ was driven accordingly to attain $100 \mathrm{MHz}$ of $\mathrm{IF} / 2$. Since most lock-in amplifiers are capable of taking reference signals with a double frequency input option, the amplitude and phase signals at ports $A$ and $R$ can be resolved using a $200 \mathrm{MHz}$ IF reference. The demodulated microwave components from the photodiode contain mainly the second-order harmonics of $\mathrm{RF} / 2$, as well as the weak fundamental components. The null DC biasing of an electro-optic modulator (i.e., to yield a minimum output at the drive frequency) can generally maintain the second-order components at a level that is at least $30 \mathrm{~dB}$ higher than the fundamental frequency. To make the second-order components more effectively dominant for the VNA signal source, a $\mathrm{Ku} / \mathrm{K}$ band amplifier was used after the photodiode.

The four complex traces in Fig. 11 are measured raw reflections with a $3.5 \mathrm{~mm}$ short-open-load calibration kit (Agilent Technologies: 85052C) and a $\mathrm{Ku} / \mathrm{K}$ band antenna. The traces are shown at a center frequency of 17 $\mathrm{GHz}$ - the strongest resonant frequency of the antenna - with a $1 \mathrm{GHz}$ span. Rather than importing the actual calibration kit data for precise error correction, we used ideal values of open $(\Gamma=1)$, short $(\Gamma=-1)$, and load $(\Gamma$ $=0$ ) for convenience. Fig. 12 is the return loss of the antenna in the $16 \sim 20 \mathrm{GHz}$ band. Here, the return loss

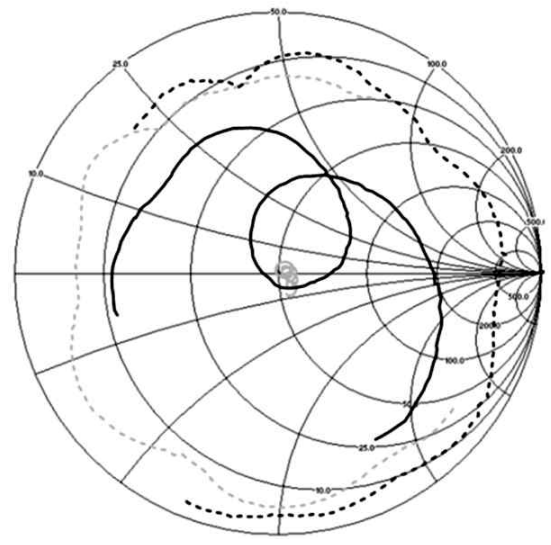

Fig. 11. Uncalibrated reflections in the $16.5 \sim 17.5 \mathrm{GHz}$ band for four terminations: Open (dashed-black), short (dashed-gray), load (solid-gray), and the antenna (solid-black).

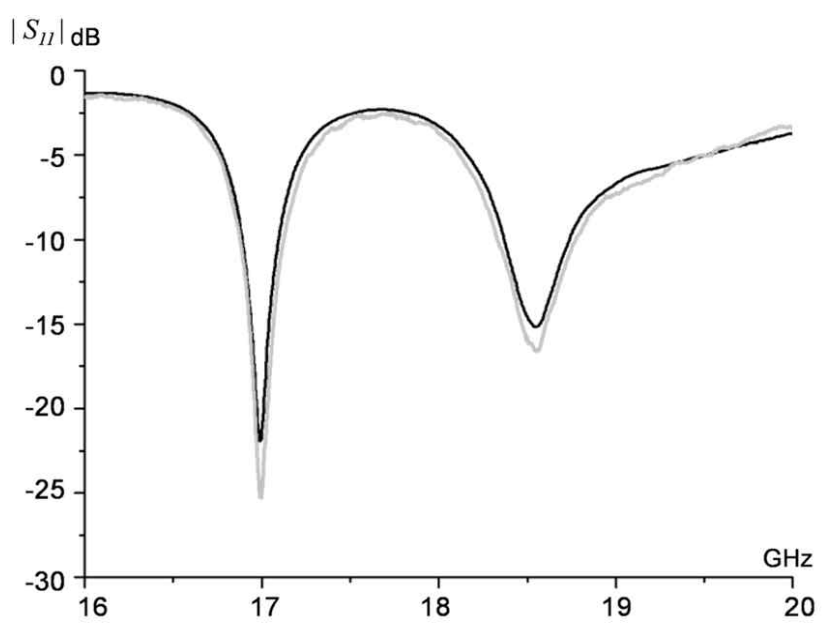

Fig. 12. Error-corrected return losses of an antenna measured with a photonic assisted system (gray) and a commercial VNA (black).

measured in our system is compared with measurements from the commercial VNA. The result generally agrees well, except at the highly resonant points. Also, our system typically shows some mild ripples, such as those observed around $17.5 \mathrm{GHz}$. This could be reduced by employing calibration kit parameters from the vendor to extract more precise error terms.

The bandwidth could be extended considerably upward to the millimeter wave range or even higher using high-frequency modulation techniques. State-of-the-art technology would enable millimeter-wave signal generation and detection, possibly up to $100 \mathrm{GHz}$ [17]. Extensions of the frequency multiplication techniques to $4 \times$ or even higher factors would be a good scheme if the frequency sweeping capability is realized. A frequency swee- 
pable, quadrupling photonic link has been implemented for VNA applications. However, it has not shown sufficiently repeatable performance as a stable VNA source because a mechanically controllable delay was used to realize the sweeping. In the near future, a VNA with higher frequency response could be made possible by stabilizing the photonic link.

\section{Conclusion}

We have demonstrated a simplified, relatively cost-effective, one-path/two-port VNA configuration with a stable optical link that can also be used for frequency multiplication of the RF signal input and local oscillator. Due to the nature of the one-path optical link, rather than using conventional bidirectional electrical VNAs, a discrete full two-port calibration scheme was used. The $S$-parameters of mismatch loads, a microwave filter, and an antenna were evaluated through conventional network analysis and several photonic-assisted methods. Harmonic frequency multiplication (at higher factors than $2 \times$ ), along with techniques for sweeping the photonic sidebands and for down-mixing the test signals to the $\mathrm{MHz}$ regime will help to extend our $20 \mathrm{GHz}$ demonstration out to the millimeter wave regime in the future.

\section{References}

[1] M. L. Van Blaricum, "Photonic systems for antenna applications," IEEE Antennas Propag. Mag., vol. 36, no. 5, pp. 30-38, Oct. 1994.

[2] S. Kurokawa, M. Hirose, and K. Komiyama, "Antenna measurements by novel optical link system using new microwave-optical technologies," in Proc. Antenna Meas. Tech. Assoc., pp. 355-360, Nov. 2005.

[3] M. Hirose, S. Kurokawa, and K. Komiyama, "Antenna measurements by one-path two-port calibration using radio-on-fiber extended port without power supply," IEEE Trans. Inst. Measure., vol. 56, no. 1, pp. 397-400, Apr. 2007.

[4] T. Nagatsuma, "Photonic measurement technologies for high-speed electronics", Meas. Sci. Technol., vol. 13, pp. 1655-1663, 2002.

[5] J. Zhang, H. Chen, M. Chen, T. Wang, and S. Xie, "Photonic generation of a millimeter-wave signal based on sextuple-frequency multiplication," Opt. Lett., vol. 32, pp. 1020-1022, May 2007.

[6] J. Zhang, H. Chen, M. Chen, T. Wang, and S. Xie, "A photonics microwave frequency quadrupler using two cascaded intensity modulators with repetitious optical carrier suppression," Phot. Technol. Lett., vol. 19, pp. 1057-1059, Jul. 2007.

[7] C. Lin, P. Shih, J. Chen, W. Xue, P. Peng, and S.
Chi, "Optical millimeter-wave signal generation using frequency quadrupling technique and no optical filtering," Phot. Technol. Lett., vol. 20, no. 21, pp. 1027-1029, Jun. 2008.

[8] M. Mohamed, X. Zhang, B. Hraimel, and K. Wu, "Frequency sixupler for millimeter-wave over fiber systems," Opt. Expr., vol. 16, pp. 10141-10151, Jul. 2008.

[9] K. Sasagawa, A. Kanno, and M. Tsuchiya, "Instantaneous visualization of K-band electric near-fields by a live electrooptic imaging system based on double sideband suppressed carrier modulation," Journal of Lightwave. Technol., vol. 26, no. 15. pp. 27822778, Aug. 2008.

[10] D. J. Lee, J. F. Whitaker, "Bandwidth enhancement of electro-optic field sensing using photonic downmixing with harmonic sidebands," Opt. Expr., vol. 16, no.19. pp. 14771-14779, Sep. 2008.

[11] J. Chen, C. Lin, P. T. Shih, W. J. Jiang, S. P. Dai, Y. M. Lin, P. C. Peng, and S. Chi, "Generation of optical millimeter-wave signals and vector formats using an integrated optical I/Q modulator," Journal of Opt. Netw., vol. 8, no. 2, pp. 188-1415, Feb. 2009.

[12] C. Lin, P. Shih, W. Jiang, E. Wong, J. Jyehong Chen, and S. Chi, "Photonic vector signal generation at microwave/millimeter-wave bands employing an optical frequency quadrupling scheme," Opt. Lett., vol. 34, no. 14, pp. 2171-32173, Jul. 2009.

[13] D. J. Lee, J. F. Whitaker, "Bandwidth enhancement of electro-optic sensing using high-even-order harmonic sidebands," Opt. Expr., vol. 17, pp. 1490914917, Aug. 2009.

[14] Y. Zhao, X. Zheng, H. Wen, and H. Zhang, "Simplified optical millimeter-wave generation configuration by frequency quadrupling using two cascaded Mach-Zehnder modulators," Opt. Lett., vol. 34, no. 21, pp. 3250-3252, Nov. 2009.

[15] S. Rehnmark, "On the calibration process of automatic network analyzer systems," IEEE Trans. Microwave Theory Tech., vol. MTT-22, no. 4, pp. 457-458, Apr. 1974

[16] S. Rehnmark, "Applying error correction to network analyzer measurements," Application Note 1287-3, Agilent Technologies, 1999.

[17] J. Li, T. Ning, L. Pei, and C. Qi, "Millimeter-wave radio over fiber system based on two-step heterodyne technique," Opt. Lett., vol. 34, no. 20, pp. 3136-3138, Oct. 2009.

[18] D. J. Lee, J. Y. Kwon, N. W. Kang, J. G. Lee, and J. F. Whitaker, "Vector-stabilized reactive nearfield imaging system," IEEE Trans. Instrum. Meas., to be published, Apr. 2011. 


\section{Dong-Joon Lee}

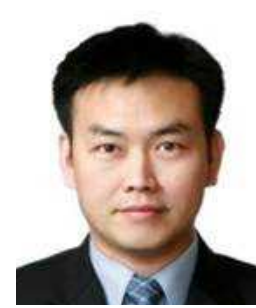

received dual B.S. degrees in physics and electronic engineering from Kon-kuk University, Seoul, Korea, in 1995 and 1996, and M.S. and Ph.D. degrees in electrical engineering from Polytechnic University, Brooklyn, N.Y. in 1999 and the University of Michigan at Ann Arbor in 2008. From 1999 to 2002, he was with the Wireless Communication Division, Samsung Electronics at Suwon, where he served as a mobile microwave design engineer. In 2009, he joined the Korea Research Institute of Standards and Science (KRISS). His research interests include the development of a high-speed electro-optic measurement technique for characterizing microwave and millimeter wave circuits and radiators, atomic-based microwave power standards, and photonicassisted microwave impedance measurement.

\section{Jae-Yong Kwon}

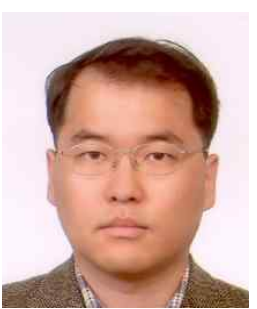

was born in Daegu, Korea in 1972. He received a B.S. degree in electronics from Kyungpook National University, Daegu, Korea in 1995 and M.S. and Ph.D. degrees in electrical engineering from the Korea Advanced Institute of Science and Technology (KAIST), Daejeon, Korea, in 1998 and 2002. From 2002 to 2005 , he was a Senior Research Engineer at the Devices and Materials Laboratory of the LG Electronics Institute of Technology. Since 2005, he has been a Senior Research Scientist in the Division of Physical Metrology, Center for Electromagnetics, Korea Research Institute of Standards and Science (KRISS), Daejeon, Korea. His research interests include electromagnetic power, impedance, and antennas.

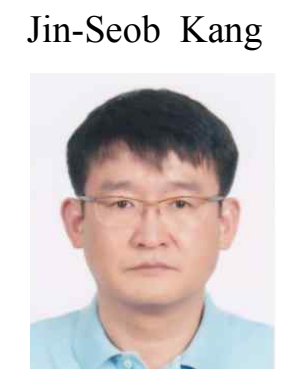

received a B.S. degree in electronic engineering from Hanyang University, Seoul, Korea, in 1987 and M.S. and Ph.D. degrees in electrical engineering from the Korea Advanced Institute of Science and Technology (KAIST), Daejeon, Korea, in 1989 and 1994. He was a Visiting Postdoctoral Research Associate in the Department of Electrical and Computer Engineering at the University of Illinois at Urbana-Champaign, USA, in 1995, and an Assistant Professor at the School of Electrical and Electronics Engineering at Chungbuk National University, Korea, from 1996 to 1997. He joined the Korea Research Institute of Standards and Science (KRISS) in 1998. His research interests include electromagnetic measurement standards, impedance and antenna measurements, and electromagnetic wave scattering.

John F. Whitaker

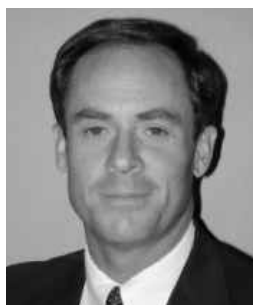

received a B.S. degree in physics from Bucknell University, Lewisburg, Pennsylvania, USA, and M.Sc. and Ph.D. degrees in electrical engineering from the University of Rochester, Rochester, New York, USA. Dr. Whitaker joined the faculty of the Department of Electrical Engineering and Computer Science at the University of Michigan in Ann Arbor in 1989 and he currently holds the titles of Research Scientist and Adjunct Professor. He has co-founded two start-up companies, Opteos, Inc. (2002) and Advanced Fiber Sensors, Inc. (2010), where he currently serves as Vice-President and Chief Technology Officer. Dr. Whitaker is the author or coauthor of more than 110 papers in journals, archival conference digests, and books and he holds two patents. Dr. Whitaker is a member of IEEE Microwave Theory and Techniques (MTT-S) and the IEEE Photonics Society, and he was a recipient of the 1996 Microwave Prize from the IEEE MTT-S. 